\title{
Statistical Analysis of Ultra Trail Marathon Organizations in Turkey
}

\author{
Andım Oben BALCE \\ Department of Econometrics \\ Pamukkale University \\ Email: abalce@pau.edu.tr \\ ORCID ID: 0000-0003-0759-1686 \\ Turkey
}

ABSTRACT

In this study, some basic statistics were calculated by using the data of the participants of Ultra trail marathon organizations. Ultra trail marathon events have been held in Turkey for 10 years. These organizations provide a significant social-economic contribution to the region where it is arranged. The data about the participants of these organizations were collected, compiled, and analyzed in this study. As a result of the study, basic data required for more comprehensive data analysis are proposed.

\section{KEYWORDS: Running, marathon, ultra trail marathon, sports participation, basic statistics}

\section{Introduction}

Statistics are used extensively in the modern world by institutions or individuals, especially in making strategic decisions. The use of statistics can appear in different names in the literature. Although the meaning of the word analytics corresponds to the processes of making and interpreting useful results in making decisions from appropriate data, it is known that analytics is heavily dependent on statistical methods. Analytics developed for businesses are named as business analytics and health-related ones such as health analytics. Sports analytics likewise defines the use of appropriate statistics to improve the performance of a team or athlete (Wikipedia). There are several types of running organizations in the world. The most popular in recent years are ultra-trail marathons organizations. These organizations in Turkey attract attention in recent years. In this study, some statistics for organizations and participants calculated from the data collected from ultratrail marathon organizations between the years of 2010 and 2019 in Turkey. Since such organizations are quite new, it is thought that such a study will benefit organizers and participants to make better decisions in future organizations. In this way, a study on data collection, compilation, and inference has not been found in the literature. Some studies that may be related to the subject in the literature are summarized below.

Andrew, Pedersen, and McEvoy (2020) describe statistical and research methods that can be used in sports management. Saaynab and Saayman (2014) examined the social and economic effects of sports organizations between 1990 and 2014. Malchrowicz-Mosko and Poczta (2018) examined small-scale sports organizations in terms of the tourism industry. Carvalho, Sousa, Paipe, Bavaresco, and Felipe (2018) conducted an economic impact analysis by examining the expenditures of half marathon participants in Portugal. Baumann and Matheson (2017) examined the effect of sports organizations held in Hawaii between 2004 and 2015 on the number of daily flights. Agrusa, Lema, Kim, and Botto (2009) investigated the economic impact of the 2007 Honolulu Marathon on the city. Corrion, Morales, Bergamaschi, Massiera, Morin, and d'Arripe-Longueville (2018) have tried to determine the psychological effects of ultramarathoners dropping outs. By using the structural equation model, Genç, Kaya, Eren, Genç, \& Y1ldırım (2019) determined the effects of the participants' participation decision on the features of the place where the running activities were performed.

Studies on the socio-economic impact of the place where running organizations are held and physical and psychological studies for the performances of the participants are widely covered in the literature.

The next part of the study that is planned as follows: Chapter 2 provides information about the development of running organizations in Turkey. In Chapter 3, explanations about the material and method followed in this study are given. Chapter 4 consists of results and some explanations about results. The last chapter is about conclusions and discussions.

\section{Running Organizations in Turkey}

Many running organizations organized and pioneered by the Turkish athletics federation have been held regularly for years. The first running or athletic race in Turkey was held on September 5, 1924, Eskisehir (Turkish Athletic Federation). The first Great Atatürk run was held in Ankara Dikmen Valley in 1936. The Eurasia Marathon, today's Istanbul marathon, was first run in 1979 (Istanbul Marathon). Marathon, half marathon, and shorter distance road races where professional athletes and people who wish to run can participate together have organized regularly in various places of Turkey, such as Mersin, Antalya, Trabzon, Kars, Gelibolu, etc.

Running race distances are classified as follows:

1. Short distances (less than $21 \mathrm{kms}$ ) 


\section{(C) Center for Promoting Education and Research (CPER) USA}

WWW.cpernet.org

2. Half marathons $(21.6 \mathrm{kms})$

3. Marathons $(42.1 \mathrm{kms})$

4. Ultramarathons (more than $42.1 \mathrm{kms}$ )

If the running event is organized mostly in terrain path conditions, whatever the race distance is, trail adjectives should be added to the name such as the Istanbul trail marathon or Istanbul Trail ultra-marathon.

Wikipedia gives some definitional, organizational, individual best records about ultramarathon organizations all over the world. In Turkey the first ultra-marathoner is a woman, her name is Bakiye Duran which is author of the book "Cesaret Yalnızdır-Bir ultra maratoncunun hikayesi" (Courage Is Alone - The story of an ultra marathonist).

There is no source about the first ultra trail marathon in Turkey. In my research, I found the first ultra-marathon is Lycian Way Ultra Marathon which is indeed a multiday running marathon in 2010 (Lycian Way Ultra Marathon). In my research, my main source is an international trail running association (ITRA) that has a list of ultra trail marathon organizations list from all over the world. I've also used each organization's internet page to get race participants and results from the information. I think here is the best place in this study to give chronological rank for ultra trail marathon organizations in Turkey.

1. 2010-Lycian Way Ultra Marathon

2. 2011-Deer Running

3. 2012-Cekmekoy Ultra Marathon

2012-Iznik Ultra Marathon

2012-Run fire Cappadocia Ultra Marathon

4. 2013-Istiklal Way Ultra Marathon

5. 2014-Cappadocia Ultra Trail Marathon

6. 2015-Aladaglar Sky Trail Marathon

2015-Kackar Ultra Marathon

2015-Tahtalı Run to Sky Trail Marathon

7. 2016-Bodrun Ultra Marathon

2016-Erciyes Sky Trail Marathon

2016-Ida Ultra Trail Marathon

2016-Manavgat Ultra Marathon

2016-Longest Night Run

2016-Taurus Challenge Marathon

8. 2017-Ephesus Ultra Marathon

2017- Ecological Artvin Ultra Marathon

2017- Phrygian Ultra Marathon

2017-Run Fire Salt Lake Ultra Trail Marathon

2017-Sapanca Ultra Marathon
2017-Uludag Ultra Trail Marathon

9. 2018-Alanya Ultra Trail Marathon

10. 2019-Istanbul Ultra Trail Marathon

2019-Latmos Ultra Marathon

2019-Kyzikos Ultra Marathon

2019-Pamukkale Ultra Marathon

There might be some ultra-marathon organizations that have internet pages or any ITRA record in Turkey. These have been excluded from the work.

In this study, available information about the

participants has been collected year by year since the first year of the organizations and useful information is explored from this data. The steps from data collection to analysis are explained in the section below.

\section{Material and Methods}

In this section, how the data is collected, compiled, and analyzed. Names and first year of ultra marathons organizations in Turkey were given above. There are 27 organizations in total and some of them continue their activities while others do not or the name has changed.

Some organizations have very well-organized internet pages that one can find race results and participant's information. The format that some organizations offer race results cannot directly be exported to excel. File types can be in the picture, pdf, word, or other formats. The basic program I use is Microsoft Excel, so all the data in these formats have been converted to Excel that is very labor-intensive work. In this study, for each race and each runner, names, ages or age categories, nationality, finished total time information was obtained in this way. However, not every organization provided all this information. Therefore, the information obtained for each organization, the participant's name, surname, gender, and completion time. As we will suggest in the conclusion and discussion section, the fact that each organization provides basic information of participations such as gender, age, countries, and cities, finish time, will facilitate those who want to do academic or organizational research in this regard. As in Itra, standard outcome disclosure information is also recommended at the end of this study.

The organizations do not have the same distances exactly. Each has different courses that have different distances. Distances are categorized as short, medium, and ultra as follows:

\begin{tabular}{l|l} 
& Distances \\
\hline Short & Less than $21 \mathrm{~km}$ \\
\hline Medium & Between $22 \mathrm{~km}$ and $42 \mathrm{~km}$ \\
\hline Ultra & More than $42 \mathrm{~km}$ \\
\hline
\end{tabular}


After the data provided by each organization was obtained and transferred/converted to excel, data manipulation was done since the names and genders of the participants from the organization to the organization were written in a different format or incomplete. Then the data transfer to the SPSS software. The data analyzes were carried out using SPSS and excel.

Since the available data is very limited (name, gender, organization year, and distance) and categorical, a descriptive and complementary analysis method has been followed with frequency distribution, table, and graphic methods. As a result of this study, if the data to be suggested about the participants are provided, researchers and organizers will be able to make good decisions by making more useful analyses that can shed light on further.

\section{Data Analysis Results}

The data we compiled from ultra-marathon organizations organized between 2010 and 2019 consists of the date of organization, distance, the name, and gender of the participants. Graphical information about the number of participants can be requested according to the most basic or first years that come to mind.

\section{(2010-2019)}

\subsection{The number of Participants for Each Gender}

The development of the number of participants year by year for each gender is important statistics for the organizers. It gives information about how the target audience has grown in terms of years. One can say from the chart below that the Turkish ultra trail marathoner's participation has grown exponentially in the last 10 years. When the increase in the number of male and female participants is examined, it is possible to say that the number of male participants increased slightly more than females. If the Covid-19 pandemic did not occur, the expectation that the number of ultra trail marathon participants would increase significantly in 2020 and later years could come true. However, it is not possible to say that the results of this pandemic will affect the running organizations positively or negatively.

\section{Graph 1. The Number of Participants for Each Gender (2010-2019)}

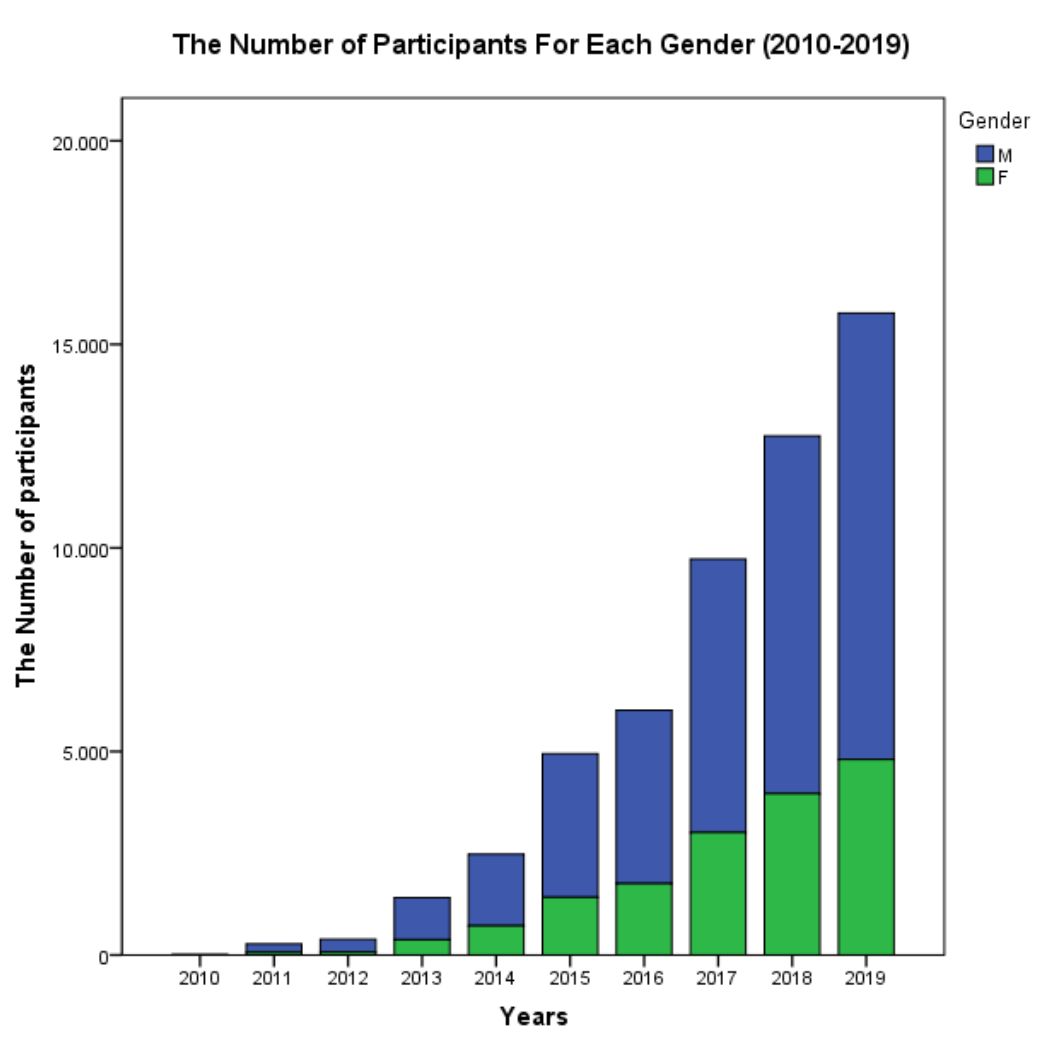

In the previous section, the list of organizations according to the first years of activity was given. It can be said that the increase in the number of organizations from 2010 to 2019 is in line with the increase in the number of participants.

\subsection{Distance Preference of the Participants}

One of the important statistics may be the distance preference of the races or the participants. As mentioned in the previous section, distances are not the same in each organization. Therefore, it has classified the distances as short, medium, and ultra. The demands for these distances by years are given in Graph 2. 


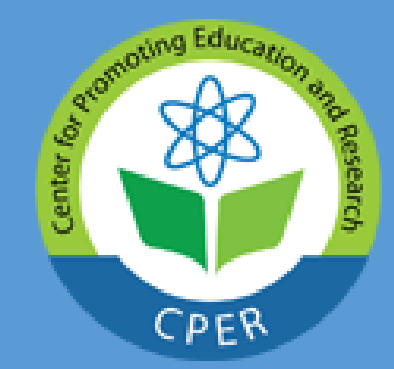

International Journal of Business and Applied Social Science (IJBASS)

E-ISSN: 2469-6501

VOL: 6, ISSUE: 7

July/2020

DOI: $10.33642 / \mathrm{ijbass.v6n7p9}$

https://iibassnet.com/

(c) Center for Promoting Education and Research (CPER) USA

WWW.cpernet.org

\section{Graph 2. Distance Preferences of the Participants by years}

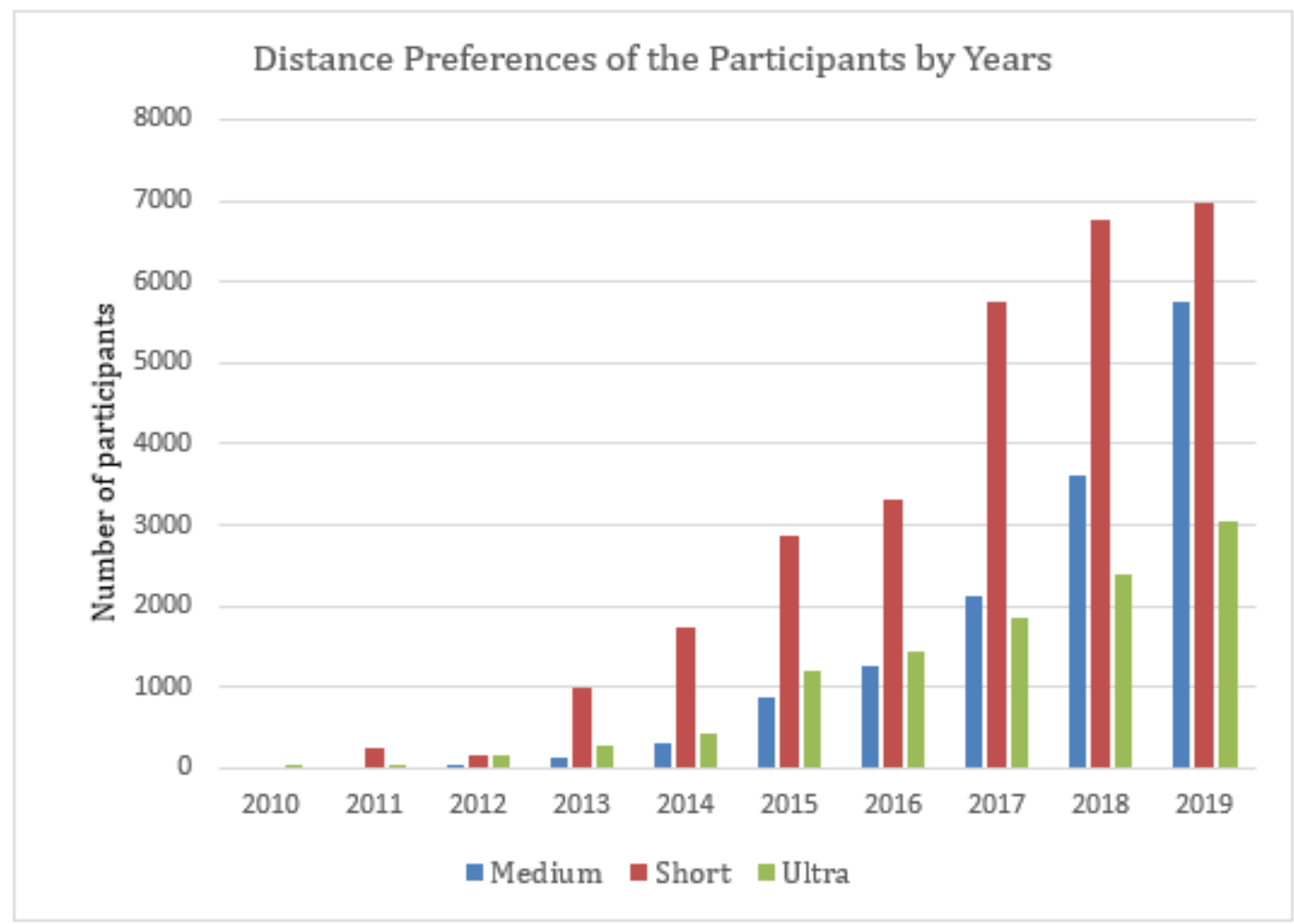

Looking at Chart 2, the demand for short and longdistance increased faster, except for long distances. It can be said that the number of long-distance runners has increased steadily and created an important demand.

Some participants go to at least one organization every year in the past 10 years. Therefore, statistics about this regular

group of runners can provide information about the future of the ultra trail marathons. The distribution of runner's preference for running at different distances for 10 years is given in the table below.

\section{Table 1. Frequency Distribution of Distance Preferences of Participants}

\begin{tabular}{lll} 
& Frequency & Percent \\
\hline Only 1 Distance & 18262 & 82,3 \\
2 Distances & 2904 & 13,1 \\
3 Distances & 1033 & 4,7 \\
Total & 22199 & 100 \\
\hline
\end{tabular}

The majority of the 22199 participants participated in types. It can be understood from the table above that the rate of the race of only 1 distance type in these 10 years. 1033 runners considering diversifying the running distance is about participants, which were considerable, competed in 3 distance $18 \%$.

Table 2. Single Distance Runners

\begin{tabular}{lll} 
& Frequency & Percent \\
\hline Short & 3490 & 19,1 \\
Medium & 12308 & 67,4 \\
Ultra & 2464 & 13,5 \\
Total & 18262 & 100 \\
\hline
\end{tabular}




\section{(C) Center for Promoting Education and Research (CPER) USA}

WWW.cpernet.org

The table above only gives information about those Some runners have participated in the ultra trail marathons for running in the same distance category. The majority of the participants prefer to run medium distances only.

4.3. Distribution of Regular Participants for Last 10 almost every year. The table below shows the frequency distribution of runners for attending at least one organization Years

Table 3. Frequency Distribution of Regular Participants

\begin{tabular}{|c|c|c|c|}
\hline & & Frequency & Percent \\
\hline \multirow{10}{*}{ 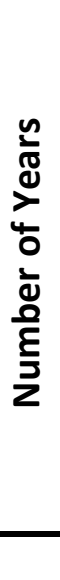 } & 1 & 15723 & 70,80 \\
\hline & 2 & 3670 & 16,50 \\
\hline & 3 & 1509 & 6,80 \\
\hline & 4 & 708 & 3,20 \\
\hline & 5 & 358 & 1,60 \\
\hline & 6 & 142 & 0,60 \\
\hline & 7 & 59 & 0,30 \\
\hline & 8 & 21 & 0,10 \\
\hline & 9 & 9 & 0,00 \\
\hline & otal & 22199 & 100 \\
\hline
\end{tabular}

Table 1 provides important information. It shows the attended at least one or two years, while the remaining $12 \%$ frequency of 22199 different participants joining the attended at least one organization in three or more years.

organizations at least once in 10 years. 15723 of the 22199

It is understood from the table above and the participants participated in at least one organization in only a histogram below that the number of regular participants have a year in 10 years. It is worth mentioning that there were only skewed structure to the right. It seems this skew will reach the one organization and 10 participants in 2010. It is observed asymmetrical structure over time, considering the increase in that approximately $88 \%$ of all ultra trail marathon participants the number of participants.

\section{Graph 3. Histogram of Regular Participants}

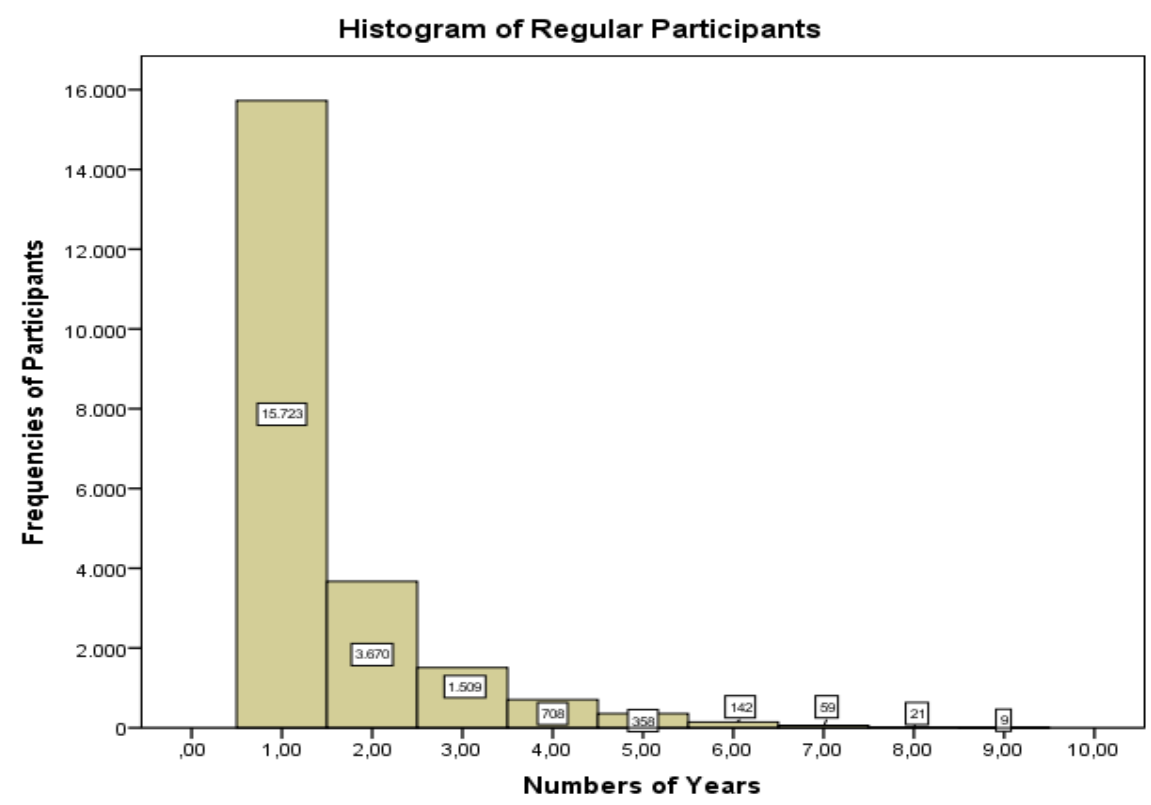

From the collected data, races were organized at 55 different $12 \%$ of the participants attended at least one organization. It is distances in 10 years. The participation distribution of the concluded from Table 2 that these participants participated in runners is given in Table 2 below. It was mentioned above that at least 5 races for 10 years. 
Table 4. Frequency Distribution of Number of Races Participated in 10 Years

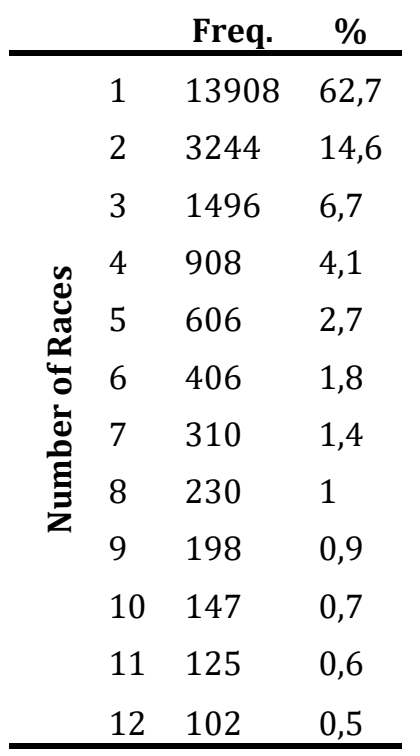

\begin{tabular}{|c|c|c|c|}
\hline & & Freq. & $\%$ \\
\hline & 13 & 63 & 0,3 \\
\hline & 14 & 72 & 0,3 \\
\hline & 15 & 59 & 0,3 \\
\hline & 16 & 47 & 0,2 \\
\hline$\ddot{\mathscr{\pi}}$ & 17 & 43 & 0,2 \\
\hline$\overline{0}$ & 18 & 30 & 0,1 \\
\hline $\bar{\Xi}$ & 19 & 26 & 0,1 \\
\hline$\Xi$ & 20 & 24 & 0,1 \\
\hline & 21 & 24 & 0,1 \\
\hline & 22 & 16 & 0,1 \\
\hline & 23 & 15 & 0,1 \\
\hline & 24 & 13 & 0,1 \\
\hline
\end{tabular}

\begin{tabular}{|c|c|c|c|}
\hline & & Freq. & $\%$ \\
\hline \multirow{12}{*}{ 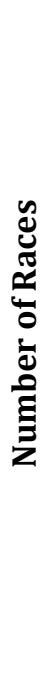 } & 25 & 20 & 0,1 \\
\hline & 26 & 8 & 0 \\
\hline & 27 & 12 & 0,1 \\
\hline & 28 & 9 & 0 \\
\hline & 29 & 3 & 0 \\
\hline & 30 & 3 & 0 \\
\hline & 31 & 1 & 0 \\
\hline & 32 & 3 & 0 \\
\hline & 33 & 2 & 0 \\
\hline & 34 & 8 & 0 \\
\hline & 35 & 2 & 0 \\
\hline & 37 & 1 & 0 \\
\hline
\end{tabular}

\begin{tabular}{|c|c|c|c|}
\hline & & Freq. & $\%$ \\
\hline \multirow{12}{*}{ 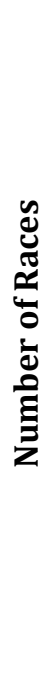 } & 38 & 2 & 0 \\
\hline & 40 & 2 & 0 \\
\hline & 41 & 2 & 0 \\
\hline & 42 & 1 & 0 \\
\hline & 43 & 1 & 0 \\
\hline & 45 & 2 & 0 \\
\hline & 46 & 1 & 0 \\
\hline & 47 & 1 & 0 \\
\hline & 49 & 1 & 0 \\
\hline & 50 & 1 & 0 \\
\hline & 55 & 1 & 0 \\
\hline & Total & 22199 & 100 \\
\hline
\end{tabular}

In general, we provided the Turkish Ultra trail

(by gender) is shown in the table below. It is observed that the number of participants has increased rapidly over the years, but due to the special situation in the country in 2016, the rate of increase in the next 1-2 years slowed down and recaptured the old increase rate in 2019 with new distances and tracks. However, how the COVID 19

Most of the ultra trail marathon events are held pandemic in 2020 will affect this rate will be seen in the every year in Turkey. One of these is selected as an example and using the data of this organization, different statistics for organizations are introduced.

future. This study also provides an opportunity to make comparisons on this subject.

This organization has been organized since 2012 . Since then, the distribution of the number of participants

Table 5. The Distribution of the Number of Participants by Gender and Years

\begin{tabular}{lllll} 
& & \multicolumn{3}{c}{ Number of Participants } \\
\cline { 3 - 5 } Years & Distances & Male & Female & Total \\
\hline \multirow{2}{*}{2012} & $\mathbf{6 0}$ & 39 & 10 & 49 \\
& $\mathbf{1 2 0}$ & 32 & 4 & 36 \\
& Total & 71 & 14 & 85 \\
\hline \multirow{2}{*}{$\mathbf{2 0 1 3}$} & $\mathbf{1 0}$ & 190 & 92 & 282 \\
& $\mathbf{4 2}$ & 73 & 29 & 102 \\
& $\mathbf{1 3 0}$ & 65 & 7 & 72 \\
& Total & 38 & 5 & 43 \\
\hline \multirow{2}{*}{$\mathbf{2 0 1 4}$} & $\mathbf{1 0}$ & 356 & 133 & 499 \\
& $\mathbf{4 2}$ & 127 & 53 & 523 \\
& $\mathbf{1 3 0}$ & 58 & 10 & 68 \\
& Total & 586 & 235 & 821 \\
\hline
\end{tabular}

\begin{tabular}{lllll} 
& & \multicolumn{3}{c}{ Number of Participants } \\
\cline { 3 - 5 } Years & Distances & Male & Female & Total \\
\hline \multirow{2}{*}{2017} & $\mathbf{5}$ & 108 & 65 & 173 \\
& $\mathbf{1 5}$ & 195 & 129 & 324 \\
& $\mathbf{5 0}$ & 193 & 38 & 231 \\
& $\mathbf{1 4 0}$ & 48 & 11 & 59 \\
& Total & 63 & 3 & 66 \\
& $\mathbf{1 5}$ & 173 & 127 & 300 \\
& $\mathbf{3 0}$ & 134 & 69 & 203 \\
$\mathbf{2 0 1 8}$ & $\mathbf{5 0}$ & 169 & 33 & 202 \\
& $\mathbf{9 0}$ & 59 & 8 & 67 \\
& $\mathbf{1 4 0}$ & 69 & $\mathbf{5}$ & 74 \\
& Total & 604 & 242 & 846 \\
\hline $\mathbf{2 0 1 9}$ & $\mathbf{5}$ & 141 & 97 & 238 \\
\hline
\end{tabular}




\begin{tabular}{lllll} 
& $\mathbf{1 0}$ & 500 & 275 & 775 \\
$\mathbf{2 0 1 5}$ & $\mathbf{4 2}$ & 178 & 62 & 240 \\
& $\mathbf{8 0}$ & 74 & 8 & 82 \\
& $\mathbf{1 3 0}$ & 77 & 7 & 84 \\
& Total & 829 & 352 & 1181 \\
\hline \multirow{4}{*}{$\mathbf{2 0 1 6}$} & 223 & 128 & 351 \\
& $\mathbf{5}$ & 111 & 56 & 167 \\
& $\mathbf{1 5}$ & 161 & 58 & 219 \\
& $\mathbf{5 0}$ & 76 & 8 & 84 \\
& $\mathbf{1 3 0}$ & 54 & 4 & 58 \\
& Total & 625 & 254 & 879 \\
\hline
\end{tabular}

\begin{tabular}{llll}
$\mathbf{2 0}$ & 219 & 155 & 374 \\
$\mathbf{3 5}$ & 141 & 45 & 186 \\
$\mathbf{5 5}$ & 163 & 28 & 191 \\
$\mathbf{9 0}$ & 66 & 4 & 70 \\
$\mathbf{1 6 0}$ & 73 & 10 & 83 \\
Total & 803 & 339 & 1142 \\
\hline Total & 4491 & 1815 & 6306
\end{tabular}

An important statistic for each organization is the loyal participants. A total of 4309 different runners number of runners who regularly participate in one of the races participated in this organization in 8 years and only 5 of them within this organization. The name of these statistics can be a can be called $100 \%$ loyal participants. 5\% of all participants loyal runner to the organization. ran for 4 or more years under this organization.

A total of 4309 different runners participated in this organization in 8 years and only 5 of them can be called $100 \%$

Table 6. The Distribution of the Loyal Runners for an Organization

\begin{tabular}{|c|c|c|c|}
\hline \multirow{9}{*}{ 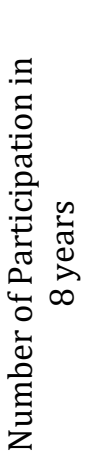 } & & Frequency & Percent \\
\hline & 1 & 3157 & 73,3 \\
\hline & 2 & 694 & 16,1 \\
\hline & 3 & 261 & 6,1 \\
\hline & 4 & 114 & 2,6 \\
\hline & 5 & 50 & 1,2 \\
\hline & 6 & 21 & 0,5 \\
\hline & 7 & 7 & 0,2 \\
\hline & 8 & 5 & 0,1 \\
\hline & Total & 4309 & 100 \\
\hline
\end{tabular}

The distribution of the course preferences of the participants in this organization is given in the table below.

Table 7. The Distribution of the Course Preferences of the Runners for an Organization

\begin{tabular}{lllllll} 
& \multicolumn{2}{c}{ Short } & \multicolumn{2}{c}{ Medium } & \multicolumn{2}{c}{ Ultra } \\
\cline { 2 - 7 } & Frequency & Percent & Frequency & Percent & Frequency & Percent \\
\hline 0 & 1443 & 33,5 & 3949 & 91,6 & 2918 & 67,7 \\
1 & 2379 & 55,2 & 331 & 7,7 & 871 & 20,2 \\
2 & 369 & 8,6 & 29 & 0,7 & 255 & 5,9 \\
3 & 91 & 2,1 & & & 135 & 3,1 \\
4 & 19 & 0,4 & & & 70 & 1,6 \\
5 & 7 & 0,2 & & & 35 & 0,8 \\
6 & 1 & 0 & & & 14 & 0,3 \\
7 & & & & & 6 & 0,1 \\
8 & & & & & 4 & 0,1 \\
Total & 4308 & 100 & 4308 & 100 & 4308 & 100 \\
\hline
\end{tabular}


When the table above is examined, it is concluded that the short courses of this organization are preferred more. At the same time, it can be said that those running on long courses attend regularly the organization for many years.

Important statistics that can be drawn from the data that can be compiled for 10 years from the Turkish ultra path marathon community are given here.

\section{Conclusion and Discussion}

Turkish ultra trail marathon organizations have been developing rapidly for the last 10 years. Many towns or city administrators believe that such organizations have significant social and economic contributions to their regions. Therefore, the importance of information about organizations has increased. In this study, available data from different sources about the Ultra Trail marathon organizations held in Turkey collected, organized, and analyzed. The results are given in the section above. These results were obtained using very limited data. Each organization presents the results in different information and formats. Even the results sent to ITRA may be incomplete. Organizations need to give participants names, surnames, age or age categories, gender, country, and city in which they live, time to arrive at aid stations, the time to complete the course. If each organization present their results this way, it will be very easy for academics or stakeholders of these organizations can draw important conclusions from this data. The interpretation of the statistics presented in this study may change according to the perspectives of the organization stakeholders. These results can contribute to realistic and important policies/strategies in terms of marketing, communication, and organization management related to the future.

\section{REFERENCES}

Agrusa, J., Lema, J. D., Kim, S. S. and Botto, T. (2009), The Impact of Consumer Behavior and Service Perceptions of a Major Sport Tourism Event, Asia Pacific Journal of Tourism Research, Vol. 14, No. 3, 267-277,

DOI: $10.1080 / 10941660903023960$

Andrew, D. P.S., Pedersen, P. M. and McEvoy, C. D. (2020), Research Methods and Design in Sport Management, second edition, Human Kinetics, Champaign, Illinois.

Baumann, R., W., and Matheson, V. A. (2017), Many happy returns? The Pro-Bowl, mega-events, and tourism in Hawaii, Tourism Economics, Volume 23 Issue 4, 788-802, DOI: 10.5367/te.2016.0562

Carvalho, M., J., Sousa, M., Paipe, G., Bavaresco, G. and Felipe, J., L., (2018), Economic Impact of the 11 th Douro Valley Half Marathon, Physical Culture And Sport Studies And Research, VOLUME LXXVIII, 41-49, DOI: 10.2478/pcssr-20180012.

Corrion K., Morales V., Bergamaschi A.,Massiera B., Morin J-B. ve d'Arripe-Longueville F., (2018) Psychosocial factors as predictors of dropout in ultra-trailers. PLoS ONE 13(11): e0206498. https://doi.org/10.1371/journal.pone.0206498

Duran B. (2010), Cesaret Yalnızdır, Optimist yayınevi, Istanbul.

Genç, V., Kaya, B., Eren, F., Genç, M., \& Yıldırım, M. S. (2019). Spor Etkinliklerine Katılım Motivasyonunun, Destinasyon İmaj1 ve Seyahat Motivasyonlarına Etkisi: Uluslararası Batman Yarı Maratonu Örneği. Journal of Current Debates in Social Sciences, 2(1), 67-77.

International trail running association (itra), https://itra.run/.

Istanbul Marathon, https://www.maraton.istanbul.

Lycian Way Ultra Marathon, https://www.likyayoluultramaratonu.com/TR/?p=Sonuclar\#2010

Malchrowicz-Mosko, E. and Poczta, J. (2018), A Small-Scale Event and a Big Impact-Is This Relationship Possible in the World of Sport? The Meaning of Heritage Sporting Events for Sustainable Development of Tourism-Experiences from Poland, Sustainability, 10, 4289,1-19; doi:10.3390/su10114289

Saayman, M. and Saayman, A. (2014), Appraisal of measuring economic impact of sport events, South African Journal for Research in Sport, 36(3):151-181.

Turkish Athletic Federation, http://www.taf.org.tr/tafgenel-bilgiler-ve-tarihce/\#1450680377234-104d4c6a-d608.

Wikipedia, Sport Analytics, https://en.wikipedia.org/wiki/Sports_analytics.

Wikipedia, Ultra marathon, https://en.wikipedia.org/wiki/Ultramarathon. 